\title{
Del Mensaje de Salamanca a la Educación Superior Inclusiva: Un Itinerario por Completar
}

\author{
Salamanca's Message to the Inclusiveness of Higher Education: \\ An Itinerary to Be Completed
}

\author{
Rafael Félix Bell * \\ Belinda Marta Lema Cachinell \\ Emma Zulay Delgado Saeteros \\ Alejandro Nicolás Lema Cachinell
}

Instituto Superior Tecnológico de Formación Profesional, Administrativa y Comercial, Ecuador

\begin{abstract}
El objetivo del presente artículo consiste en reflexionar acerca de la comprensión y el carácter de la educación superior inclusiva, en lo referido a las personas con discapacidad, y su desarrollo en el Instituto Superior Tecnológico de Formación Profesional, Administrativa y Comercial (ITF) de Guayaquil, Ecuador, como expresión de la respuesta a la necesidad de avanzar hacia el cumplimiento de los objetivos de este complejo proceso, completando de esa manera el itinerario delineado en Salamanca hace 25 años. Con la finalidad de dar cumplimiento al objetivo propuesto, se desarrolló una investigación con un enfoque mixto, para cuyo desarrollo se aplicaron métodos científicos de nivel teórico y empírico, que se emplearon en la revisión bibliográfica narrativa y en la aplicación de técnicas indirectas basadas en la recolección y análisis de datos extraídos de registros oficiales y reportes documentados del ITF, con el ánimo de aportar elementos para avalar el establecimiento de las dos sub-etapas por las que ha transitado y transita en la actualidad la educación superior inclusiva en el Instituto en la materialización del cumplimiento de su responsabilidad social, que resulta coherente con la indispensable y continua renovación y enriquecimiento de los propósitos de Salamanca en esta institución de educación superior.
\end{abstract}

Descriptores: Enseñanza superior; Derecho a la educación; Igualdad de oportunidades; Inclusión; Ecuador.

The purpose of this article is to ponder on the comprehension along with the nature of Inclusive Higher Learning in relation to people with disabilities within the "Instituto Superior Tecnológico de Formacion Profesional, Administrativa y Comercial (ITF)" from the city of Guayaquil. Expressing the answer in regards to the need to advance towards the fulfillment of these complex objectives, in that way completing the itinerary outlined by Salamanca 25 years ago. With the purpose of fulfilling the established goal, we developed a research with a mixed approach, for its development there was scientific methods at a theoretical and empirical level that was applied, these were used for the narrative bibliography review and the applying of indirect techniques based on the collection of data extracted from official records from the ITF, with the aim of providing elements to support the establishment of the two sub-stages, whom have been busy in today's Inclusiveness of Higher Learning within the Institute, in the achievement of the goal its socially responsible for, that result with the indispensable, an ongoing renovation and enrichment of all of Salamanca's in the Institute of Higher Learning.

Keywords: Higher education; Right to education; Equal opportunity; Inclusion; Ecuador.

*Contacto: rafael.bell@formacion.edu.ec

ISSN: 0718-7378

www.rinace.net/rlei/
Recibido: $\quad 15 / 08 / 2019$

$1^{a}$ Evaluación: 20/09/2019

Aceptado: $\quad$ 10/10/2019 


\section{Introducción}

En el numeral 48 del apartado C del marco de acción de la Declaración de Salamanca (1994) aparecen dos referencias a las universidades, específicamente, en el epígrafe titulado Contratación y Formación del Personal Docente. En el mismo, se resalta el papel consultivo de las instituciones universitarias y la necesidad que, desde ellas, se fomente la investigación en estrecho vínculo con la capacitación para contribuir al logro de los objetivos y compromisos aprobados en esa ciudad española por los representantes de 92 gobiernos y 25 organizaciones internacionales en el mes de junio de 1994.

Las escasas referencias a las universidades y la ausencia de alusión alguna a la educación superior en los citados documentos responden a las finalidades y objetivos de la conferencia en la que fueron aprobados, cuyo foco de atención fue la educación básica, la promoción y el logro de la educación para todos, con énfasis en el alumnado con necesidades educativas especiales.

Sin embargo, con el paso de los años y como resultado de los avances en la aplicación de políticas educativas alineadas a los objetivos de la Declaración de Salamanca, sus efectos se comenzaron a percibir también en la educación superior, poniendo a prueba la verdadera y plena articulación del sistema educativo, lo que presupone la conexión y las posibilidades del tránsito fluido de los estudiantes por sus distintos niveles y subsistemas.

Consiguientemente, la proclamación de la educación inclusiva como un principio de la educación no podía limitar su alcance a determinados eslabones del sistema de educación, sino que requería su reconocimiento y valoración, como apuntan Escudero y Martínez (2011, p. 88) "como un derecho esencial que ha de garantizarse a todas las personas, sin ningún género de discriminación o exclusión, es un valor y un principio fundamental, abiertamente ideológico, no fáctico”.

Todo ello dictaba la necesidad de completar en la educación superior el itinerario que tuvo su principal punto de partida en Salamanca y cuyo recorrido parece plantear una travesía interminable hacia la conquista del máximo desarrollo posible de las potencialidades de todos los estudiantes, incluidos quienes presentan alguna discapacidad y se forman como profesionales en distintas Instituciones de Educación Superior (IES).

En ese contexto, la Declaración Mundial para la Educación Superior de la UNESCO (1998), que retoma el espíritu de la Declaración Universal de los Derechos Humanos (ONU, 1948) y con posterioridad la Convención Internacional sobre los Derechos de las Personas con Discapacidad (ONU, 2006), se convirtieron en los sustentos normativos capaces de contribuir a forjar una nueva visión de la educación universitaria orientada a la promoción de las transformaciones que aseguren "que las personas con discapacidad accedan -sin discriminación y en igualdad de condiciones al resto- a la educación superior, a la formación profesional, a la educación para adultos y al aprendizaje a lo largo de la vida”(Moriña, Cortés y Molina, 2015, p. 163).

Desde esa perspectiva, considerando el paulatino crecimiento actual y perspectivo de la matrícula de estudiantes con discapacidad en distintos escenarios de aprendizaje de la educación superior, como es el caso del Instituto Superior Tecnológico de Formación Profesional Administrativa y Comercial (ITF) de Guayaquil, Ecuador, el objetivo del presente artículo consiste en reflexionar acerca de la comprensión y el carácter de la educación superior inclusiva y su desarrollo en el ITF como expresión de la respuesta a la 
necesidad de avanzar hacia el cumplimiento de los objetivos de este complejo proceso, completando de esa manera el itinerario delineado en Salamanca hace 25 años.

\section{Fundamentación teórica}

\subsection{Comprensión de la educación superior inclusiva}

Tal y como ocurre con la definición de educación inclusiva en general, de la que con posterioridad a la Declaración de Salamanca (1994) se han generado múltiples formulaciones e interpretaciones, la situación referida a la educación superior inclusiva también se caracteriza por diversidad de enfoques, perspectivas, propuestas conceptuales y formas de aplicación.

En este marco, Moriña (2004) citada por Ocampo (2013, p. 228) indica que "la educación superior inclusiva constituye un proceso orientado a proporcionar una respuesta apropiada a la diversidad de características y necesidades educativo-formativas del alumnado".

Acevedo (2015) aborda la comprensión de la educación superior inclusiva como una estrategia vital para alcanzar el cumplimiento de la misión de las IES y el logro de los objetivos de la inclusión social, poniendo a prueba el nivel de respuesta de las instituciones para propiciar el máximo desarrollo posible de las capacidades subjetivas de sus estudiantes a partir del reconocimiento de su diversidad.

Según Maltseva y Boyko (2017), la educación superior inclusiva representa una nueva etapa de desarrollo del sistema general de educación, que presupone no sólo la participación en el proceso de aprendizaje y el desarrollo intelectual de las personas con discapacidad, sino también su desarrollo socio-profesional como premisa para contribuir al bienestar de la sociedad.

En síntesis, las definiciones esbozadas con anterioridad reflejan la esencia de la educación superior inclusiva como proceso o estrategia, pero siempre orientada a promover la formación profesional de nivel superior de la diversidad del estudiantado, incluidas las personas que presentan alguna discapacidad.

Precisamente en la solución de la tensión que se genera entre las particularidades de las necesidades educativas que se presentan en algunos estudiantes y las exigencias que plantean la formación y el desarrollo de las competencias del futuro profesional radica uno de los principales desafíos de la educación superior inclusiva, que resulta mayor en la misma medida en que las condiciones que generan las referidas necesidades educativas sean más complejas y su comportamiento en el tiempo se haga más prolongado.

\subsection{Carácter sistémico de la educación inclusiva}

De acuerdo con Rubtsova (2018), uno de los criterios fundamentales en la concepción y organización de los sistemas educativos es su estructura en forma de red, en la que se articulan sus distintos subsistemas y niveles para conformar la trayectoria educativa que cada persona ha de recorrer en distintas etapas de su vida.

El referido criterio encuentra un desarrollo normativo superior al ser asumido y refrendado en la legislación vigente en cada país, como Ecuador, donde en el artículo 351 de la Constitución de la República (2008, p. 162) se consagra lo siguiente: 


\begin{abstract}
El sistema de educación superior estará articulado al sistema nacional de educación y al Plan Nacional de Desarrollo; la ley establecerá los mecanismos de coordinación del sistema de educación superior con la Función Ejecutiva. Este sistema se regirá por los principios de autonomía responsable, cogobierno, igualdad de oportunidades, calidad, pertinencia, integralidad, autodeterminación para la producción del pensamiento y conocimiento, en el marco del diálogo de saberes, pensamiento universal y producción científica tecnológica global.
\end{abstract}

Esta estructuración y articulación en red constituye un aspecto de vital importancia en el diseño y aplicación de las distintas políticas y principios educativos, pues debido a la interconexión existente entre los diferentes eslabones de la red educativa, las decisiones y cambios que se adopten en cada uno de ellos, repercutirán siempre, en mayor o menor medida, en otros.

Así, la educación inclusiva, cuyo mensaje a partir de la Declaración de Salamanca comenzó a calar cada vez con mayor profundidad en los distintos niveles de la educación básica, especialmente en la educación primaria, empezó a generar la necesidad de su aplicación en los niveles educativos subsiguientes, incluido el universitario, en los que la incorporación de estudiantes con discapacidad reclamaba una respuesta que no supusiera la negación de lo alcanzado con la gradual implementación de lo proclamado en Salamanca.

En ese camino resultaba esencial el desplazamiento del centro de los debates de las exhortaciones y fundamentos filosóficos, antropológicos, sociológicos, jurídicos, psicológicos y pedagógicos (Pérez Serrano, 2015) para avalar y acometer la inclusión, hacia las vías, métodos y procedimientos para lograr su realización en la práctica, que Loreman (2007) identifica como el paso del por qué al cómo.

A tal fin es indispensable garantizar las transformaciones en todas las esferas y ámbitos de la vida universitaria, dentro de los cuales resultan decisivos los siguientes:

- Componentes del ciclo para la educación inclusiva en la educación superior: transición/ingreso, permanencia con calidad, egreso efectivo, titulación y seguimiento.

- Procesos sustantivos de la educación superior: docencia, investigación y vinculación con la sociedad.

- Gestión. Generación de una cultura organizacional inclusiva.

- Infraestructura. Eliminación de todo tipo de barreras.

- Aspectos organizativos: agrupamientos, diversidad de modalidades y horarios de tutorías.

- Componentes didácticos: contenidos, metodologías, utilización de las tecnologías y evaluación.

- Formación inicial y permanente del profesorado.

- Subjetividad e intersubjetividad de los distintos agentes que intervienen en el proceso educativo.

La naturaleza y alcance de la educación inclusiva reflejada en las transformaciones en cada uno de los referidos ámbitos, revelan su carácter sistémico y asociado al mismo, su enorme capacidad potencial para propiciar un verdadero cambio de la educación superior que ha de estar orientado a no dejar inconcluso el itinerario propuesto en Salamanca. Indudablemente se trata de un proceso complejo, en el que las IES están llamadas no solo 
a desempeñar un papel consultivo y de promoción de la investigación, como fue declarado hace 25 años, sino también a convertirse en modelos reales de la educación inclusiva en el eslabón más alto del sistema educativo y a generar, de esa manera, un impacto de gran trascendencia laboral y social. En consecuencia, el desarrollo de la educación inclusiva presupone una transformación cualitativa planificada de todo el sistema educativo, incluida la educación superior, dirigida a dar respuesta a las más diversas y cambiantes necesidades educativas de todos los estudiantes (Zinevich, Degtyareva y Degtyareva, 2016). Lógicamente la referida transformación no se produce de manera inmediata ni resulta homogénea. En cada país este proceso, si bien comparte principios y presupuestos comunes, tiene características particulares determinadas por factores políticos, económicos, sociales y culturales, que se comportan de un modo diferente en distintos contextos.

\subsection{La educación superior inclusiva en Ecuador}

En Ecuador, como ocurre en prácticamente todos los países, el marco legal de la educación superior inclusiva en relación con las personas con discapacidad, forma parte del ordenamiento jurídico y político del país, encontrando sus principales sustentos en la Constitución de la República (2008), en la Ley Orgánica de Educación Superior (2010), en la Ley Orgánica de Discapacidades (2012) así como en la Ley Orgánica de los Consejos Nacionales para la Igualdad (2014).

Los preceptos constitucionales, las políticas y directrices que emanan de los citados cuerpos legales confirman la existencia de un sólido marco para el reconocimiento y respeto de los derechos de las personas con discapacidad, pero la situación real de una parte importante de esta población requiere la transformación definitiva de los derechos que se proclaman en hechos que se plasmen en la vida.

La brecha existente entre los derechos consagrados y los hechos llamados a confirmar su pleno ejercicio, también está presente en la educación superior ecuatoriana, en la que, de acuerdo con Herdoiza (2015) apoyada en datos del INEC (2010), es muy bajo el porcentaje de personas con discapacidad que logran ingresar y concluir sus estudios de tercer nivel $(6 \%)$ y es todavía inferior el porcentaje de aquellos que alcanzan un título de cuarto nivel $(0,5 \%)$.

A propósito de este último aspecto, Ocampo (2016, p. 67) apunta lo siguiente: "los estudios de postgrado para PsD (personas en situación de discapacidad) representan un campo de trabajo escasamente explorado por la comunidad científica y profesional especializada, en materia de educación universitaria de tipo inclusiva".

Como reflejo de ello, son muy pocas las personas con discapacidad que se desempeñan en calidad de profesionales universitarios, cuestión que también se hace evidente en los claustros de la educación superior, incluidas aquellas IES en las que se desarrollan carreras de grado y programas de maestrías relacionados con la atención a la diversidad y la educación inclusiva y entre cuyos profesores de manera muy excepcional se encuentra algún profesional con discapacidad, que de acceder por méritos propios a esos colectivos docentes, con su activa participación brindaría un ejemplo de enorme trascendencia académica, educativa y social. Desde la perspectiva de la diversidad, ello también aplica a los representantes de pueblos indígenas, afrodescendientes y de otros grupos históricamente excluidos. 
Esta última aspiración, como otras muchas que se derivan del mensaje de Salamanca, tal vez se ubique dentro de los ideales de la inclusión que hoy pueden parecer difíciles de lograr o fuera de alcance inmediato (Escudero y Martínez, 2011), pero de todos modos no se debe dejar de trabajar y apostar por su cumplimiento, empeñándose en la superación de las barreras que obstaculizan la inclusión en el nivel universitario y en la remoción de las "restricciones de tipo económico, social, técnico y político que limitan profundamente el acceso a la educación superior de las personas con discapacidad” (Espinosa, Gómez y Cañedo, 2012, p. 33).

Las barreras y limitaciones antes mencionadas se manifiestan de diversas maneras en la educación superior ecuatoriana, en cuyo contexto a veces el trabajo para el logro de los propósitos de la inclusión se reduce básicamente al cumplimiento del sistema de becas y al ofrecimiento de determinadas ayudas con la intención de favorecer el acceso y la permanencia de los estudiantes con discapacidad en la educación superior.

En ese sentido, las investigaciones consultadas, realizadas en IES ecuatorianas (Espinosa, Gómez y Cañedo, 2012; Esteves y Santos, 2014; Santiesteban et al., 2017) permiten resaltar la diversidad de factores que atentan contra el cumplimiento de los propósitos de la educación superior inclusiva, entre los cuales se incluyen los siguientes: carencia o desconocimiento de la existencia de servicios o programas de apoyo y acompañamiento a los estudiantes con discapacidad en su trayectoria educativa universitaria, persistencia de barreras que limitan la accesibilidad universal, escasa utilización de las funcionalidades de las TIC para favorecer el pleno acceso a la información, déficits de estudios e investigaciones sobre educación superior inclusiva, falta de integralidad y coherencia de las acciones que se acometen para garantizar la inclusión y las limitaciones en la preparación del claustro docente para desarrollar un proceso de enseñanza aprendizaje inclusivo.

No hay que obviar que, dado que los esfuerzos que se vienen desplegando para el logro de la educación superior inclusiva son todavía recientes (Bell, 2017; Herdoíza, 2015), no se cuenta con una tradición de inclusión en las IES, lo que genera dudas y cuestionamientos acerca de las posibilidades de éxito de tan complejo proceso en el nivel universitario en el que ha prevalecido y todavía sigue presente el rigor en la selección para el ingreso, que se expresa en la ponderación de los méritos, especialmente los de tipo académico y no se consideran en la misma medida las potencialidades, el interés, el compromiso y la perseverancia que las personas con discapacidad muestran para alcanzar diferentes metas en la vida (Santiesteban et al., 2017), lo que se maximiza en caso de contar con las ayudas y apoyos requeridos.

En medio de ese complejo panorama, una posición particular la ocupan los Institutos Superiores Técnicos y Tecnológicos, que son tal vez la evidencia más palpable de la etapa de transición por la que atraviesa la educación superior ecuatoriana (Toscanini, Aguilar Guzmán y García Sánchez, 2016), que ha de encontrar en la educación inclusiva un poderoso resorte para concretar sus propósitos.

\subsection{Renovación de la vigencia del mensaje de Salamanca en el ITF}

$\mathrm{Al}$ hilo de lo apuntado al término del apartado anterior, cabe resaltar que la aprobación de la Ley Orgánica Reformatoria de la Ley Orgánica de Educación Superior, en julio de 2018, constituye una clara expresión de la inaplazable necesidad de continuar transformando integralmente la educación superior ecuatoriana, uno de cuyos énfasis recae en el 
redimensionamiento y la revalorización de los Institutos Superiores Técnicos y Tecnológicos.

Las características de estas instituciones, entre las que sobresalen su manifiesta orientación práctica, su estrecha vinculación con la utilización de las posibilidades de las TIC, la preparación de sus graduados para acceder al mercado laboral en menor tiempo unido al creciente reconocimiento de la pertinencia e importancia de la formación técnicotecnológica, hacen que sea cada vez mayor el interés que suscitan las carreras de este perfil en diferentes sectores de la población, del que también forman parte las personas con discapacidad.

Por consiguiente, el desafío de la educación inclusiva comenzó a hacerse presente, cada vez con más fuerza, en estas IES que asumieron el compromiso de completar un itinerario que continúa teniendo en Salamanca un punto de obligatoria referencia. Ese es el caso del Instituto Superior Tecnológico de Formación Profesional Administrativa y Comercial (ITF), de Guayaquil, una institución que tiene como misión la formación de profesionales y ciudadanos con responsabilidad social y ambiental, capaces de diseñar, ejecutar y evaluar procesos relacionados con la actividad productiva e innovación tecnológica.

Cabe significar que, en la proyección de su misión, la educación inclusiva es comprendida también como uno de los modos de materialización del cumplimiento de la responsabilidad social del ITF, lo que implica, en línea con lo señalado por Navarros y Luque (2017, p. 22) "un proceso permanente de mejora continua; el camino hacia un horizonte que nunca se puede alcanzar en su totalidad".

En ese empeño, en la labor inclusiva acometida por el ITF a partir del año 2008, que marca el inicio de su actual etapa de desarrollo, se pueden distinguir dos sub-etapas fundamentales; la primera, que abarca los años del 2008 al 2014 y la segunda, que se desarrolla a partir del año 2015 hasta la actualidad.

Para el establecimiento de esta etapa con sus respectivas sub-etapas fueron considerados diferentes criterios con ajuste a lo señalado por Hernández Fernández y Pérez Véliz (2013, p. 444), quienes precisan que:

\begin{abstract}
Al considerar una etapa debe valorarse que la misma no constituye un trayecto temporal lineal, en el operan procesos de continuidad y discontinuidad, de saltos que no llegan a significar rupturas absolutas. A tales saltos y discontinuidades, los asumimos en calidad de sub-etapas, o momentos significativos dentro de una etapa determinada.
\end{abstract}

En el caso de la trayectoria inclusiva del ITF, la primera sub-etapa, que puede ser ubicada aproximadamente entre los años 2008-2014, se caracteriza por la creación de las bases para impulsar la educación inclusiva, para lo cual se trabajó en la eliminación de barreras arquitectónicas, en la colocación de señaléticas en sistema Braille y en la preparación inicial del profesorado y de toda la comunidad educativa para el trabajo con estudiantes con discapacidad.

La segunda sub-etapa, que comenzó alrededor del año 2015, se caracteriza por la profundización del trabajo en torno a la educación inclusiva, lo que se refleja en los siguientes aspectos: sistematización de la preparación pedagógica, didáctica y metodológica del profesorado para la atención a la diversidad del estudiantado, la incorporación de un mayor número de estudiantes con discapacidad al ITF, el desarrollo de herramientas metodológicas para la inclusión entre las que se destacan la generación de comunidades de apoyo y las tutorías entre iguales (mentoring), la presentación de 
ponencias y la publicación de artículos relacionados con las experiencias de educación inclusiva en el instituto en revistas nacionales e internacionales, la activa participación de los estudiantes con discapacidad en programas y actividades de vinculación con la sociedad así como la incorporación de problemáticas relacionadas con la discapacidad como temas de los trabajos de titulación de los futuros tecnólogos desde la perspectiva de las diferentes carreras que conforman la oferta académica institucional.

Derivado de la experiencia acumulada en toda esta etapa y luego del correspondiente estudio de pertinencia, el ITF elaboró y presentó el proyecto de la carrera Tecnología Superior en Educación Inclusiva, aprobada por el Consejo de Educación Superior del Ecuador mediante la resolución RPC-SO-03-NO.O49-20 L9 del 23 de enero del 2019. La aprobación de esta carrera marca un importante hito en el desarrollo de la labor inclusiva del Instituto de Formación, generando las condiciones para contribuir a la formación del talento humano capaz de asumir con compromiso el legado de Salamanca a fin de hacer posible la educación inclusiva a una mayor escala.

\section{Método}

La orientación del desarrollo del presente estudio estuvo guiada por la siguiente pregunta de investigación: a la luz del mensaje de Salamanca, ¿cuáles han sido las etapas por las que ha transitado el desarrollo de la educación superior inclusiva en relación con las personas con discapacidad en el Instituto Superior Tecnológico de Formación?

Para la búsqueda de la respuesta a la pregunta de investigación planteada se realizó un estudio con un enfoque mixto, para cuyo desarrollo se aplicaron métodos científicos de nivel teórico y empírico. Los métodos teóricos, en particular el análisis y la síntesis, la inducción-deducción y el método histórico lógico se emplearon para la revisión bibliográfica narrativa que aportó los principales referentes que fueron considerados en la elaboración del marco teórico de la investigación.

La revisión bibliográfica narrativa se realizó con la finalidad de resumir e informar acerca de diferentes aproximaciones a la comprensión y carácter sistémico de la educación superior inclusiva, precisando algunas características de la situación actual de este proceso en Ecuador como punto de partida para el esbozo de la experiencia inclusiva del ITF y su relación con la renovación de la vigencia del mensaje de Salamanca.

En esta primera etapa del estudio, dirigida a la construcción del marco teórico, fueron identificadas 32 fuentes de interés de acuerdo con el descriptor "educación superior inclusiva”, que fue asumido como principal criterio para la selección, se concentró en 19 $(59,37 \%)$, de las cuales, 5 (26,31\%) corresponden al período entre el 2007 y el 2014, mientras $14(73,68 \%)$ se publicaron desde el 2015 hasta el 2018, lo que evidencia el nivel de actualidad de las fuentes consultadas, que de acuerdo con las lenguas en las que fueron publicadas se distribuyen de la siguiente manera: 15 en español, 3 en ruso y 1 en inglés.

Junto con la revisión bibliográfica se procedió a la aplicación de técnicas indirectas basadas en la recolección y análisis de datos extraídos de registros oficiales y reportes documentados del ITF, con el ánimo de aportar elementos para avalar el establecimiento de las dos sub-etapas por las que ha transitado y transita en la actualidad la educación superior inclusiva en el Instituto de Formación. 
Para la obtención de los datos se establecieron las dimensiones e indicadores que se detallan en el cuadro 1.

Cuadro 1. Dimensiones e indicadores

\begin{tabular}{ll}
\hline \multicolumn{1}{c}{ DIMENSIONES } & INDICADORES \\
\hline & Matrícula y permanencia de estudiantes con discapacidad. \\
& Estudiantes con discapacidad graduados. \\
& Preparación del profesorado para la educación inclusiva. \\
Docencia & Incorporación de resultados de experiencias y metodologías de \\
& educación inclusiva utilizadas en el ITF para el enriquecimiento de \\
& contenidos de diversas materias de las mallas curriculares. \\
\hline & Publicación de artículos científicos referidos a la educación superior \\
& inclusiva. \\
Ponencias referidas a la educación superior inclusiva presentadas en \\
eventos. \\
Trabajos de titulación de los graduados que guardan relación con la \\
educación superior inclusiva. \\
\hline \multirow{2}{*}{ Vinculación con la } \\
sociedad & con discapacidad. \\
& Estudiantes con discapacidad que realizaron sus prácticas pre- \\
& profesionales en el ITF.
\end{tabular}

Fuente: Elaboración propia.

Los criterios para el análisis de estos indicadores se basaron en el año de generación de cada dato correspondiente a las dimensiones establecidas, que reflejan diferentes aristas relacionadas con los procesos sustantivos de la educación superior, es decir, docencia, investigación y vinculación con la sociedad, siempre desde la perspectiva de la educación superior inclusiva en lo referido a las personas con discapacidad.

\section{Resultados}

La realización de la investigación posibilitó la identificación de los elementos que permitieron establecer que en la evolución de la labor inclusiva del ITF se distinguen dos sub-etapas, la primera, que abarca los años del 2008 al 2014 y la segunda, que se desarrolla a partir del año 2015 hasta la actualidad.

A continuación se presentan los resultados del comportamiento de los indicadores establecidos en cada sub-etapa:

\subsection{Dimensión: Docencia}

Indicador: Matrícula y permanencia de estudiantes con discapacidad.

En la primera sub-etapa (2008-2014) tres estudiantes con discapacidad formaron parte de la matrícula del ITF, mientras que en la segunda sub-etapa (2015-actualidad) esa cantidad alcanzó los 29, lo que representa un incremento de 26 estudiantes. Todos los estudiantes con discapacidad permanecen en el instituto, transitando por los distintos períodos académicos.

El comportamiento de este indicador, y en particular el hecho de que el $100 \%$ de los estudiantes con discapacidad desarrolle una trayectoria académica positiva es un claro reflejo de la efectividad de las estrategias, recursos y apoyos disponibles en el ITF para evitar la generación de nuevas barreras y superar las que han sido identificadas a fin de promover el aprendizaje exitoso de la diversidad del estudiantado. 
Dimensión: Docencia

Indicador: Estudiantes con discapacidad graduados.

En la sub-etapa del 2008 al 2014 se graduaron 3 estudiantes con discapacidad, cantidad que se elevó a 11 en la actual sub-etapa.

La graduación de los estudiantes con discapacidad, en particular de los 11 que se incorporaron como tecnólogos superiores en la actual sub-etapa, ha permitido corroborar la validez de la estrategia de titulación aplicada en el Instituto, entre cuyas acciones se destaca el desarrollo de sesiones colectivas de orientación, el papel de la mentoría entre iguales y las tutorías individualizadas de investigación.

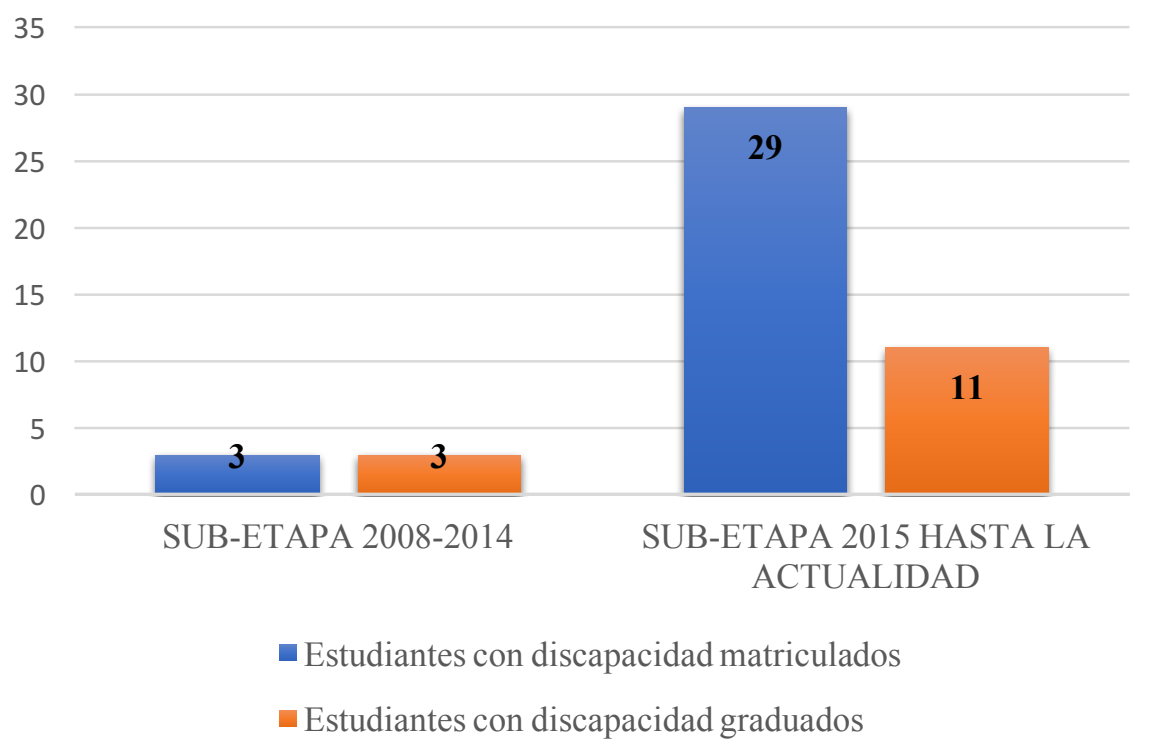

Figura 1. Matrícula y estudiantes con discapacidad graduados Fuente. Elaboración propia.

Dimensión: Docencia

Indicador: Preparación del profesorado para la educación inclusiva.

La valoración de este indicador se realizó considerando la aplicación en la práctica educativa de las competencias desarrolladas por los docentes durante los talleres y jornadas de capacitación para la educación inclusiva realizados en cada sub-etapa.

Cabe significar que en la primera sub-etapa se identificaron escasas evidencias de aplicación práctica de las competencias del profesorado para la inclusión, en tanto en la segunda sub-etapa, el análisis de los resultados de las observaciones de clases, de la producción científica del instituto, de las actas de las reuniones generales de docentes y de los colectivos académicos de carrera como parte del sistema de acompañamiento metodológico vigente, revela la aplicación de estrategias y herramientas para la inclusión, entre las que sobresalen el establecimiento del expediente psicopedagógico, la generación de comunidades de apoyo en los cursos, el trabajo de orientación con la familia, el fortalecimiento de las tutorías educativas y académicas, la realización de determinadas adaptaciones curriculares no significativas en respuesta a las necesidades educativas de los estudiantes. 
Dimensión: Docencia.

Indicador: Incorporación de resultados de experiencias y metodologías de educación inclusiva utilizadas en el ITF para el enriquecimiento de contenidos de diversas materias de las mallas curriculares.

Los resultados del desarrollo de experiencias y de la aplicación de metodologías de educación inclusiva utilizadas en el ITF, en particular, del año 2015 hasta la actualidad, contribuyeron, como se evidenció en el análisis de los registros del diseño y rediseño curricular, al enriquecimiento de contenidos curriculares en diversas materias, entre las que se incluyen, por citar dos ejemplos Comunicación oral y escrita y Ética profesional y responsabilidad social.

Además, esos resultados fueron de utilidad para el enriquecimiento de contenidos de materias impartidas en el 4to. nivel, como son Problemas Contemporáneos de la Inclusión Educativa y la Atención a la Diversidad y Atención educativa a la diversidad sensorial en Instituciones Inclusivas, que se imparten como parte de la malla de la maestría en Educación Mención en Educación Inclusiva y Atención a la Diversidad.

\subsection{Dimensión: Investigación}

Indicador: Publicación de artículos científicos referidos a la educación superior inclusiva.

Antes del año 2015, que marca el inicio de la segunda sub-etapa, no aparecen registros de publicaciones de artículos científicos relacionados la educación superior inclusiva elaborados por docentes del ITF. A partir de ese año, este tema comienza a ser tratado con sistematicidad y desde diversas aristas, como lo reflejan los 10 artículos publicados en esta sub-etapa, que han sido editados por prestigiosas revistas nacionales e internacionales, entre las que se incluyen la Revista Latinoamericana de Educación Inclusiva (Chile), PublicacionesDidácticas (España) y la Revista Investigación, Formación y Desarrollo: Generando productividad institucional (Ecuador).

Dimensión: Investigación

Indicador: Ponencias referidas a la educación superior inclusiva presentadas en eventos.

En la primera sub-etapa no se encontraron evidencias de la presentación, por profesores del ITF, de ponencias vinculadas con la educación superior inclusiva en jornadas, reuniones científicas y congresos. Desde el año 2015 se constató una transformación en este indicador, con un total de 15 ponencias presentadas en diferentes eventos, entre los que sobresalen los Congresos Internacionales de Ciencias Pedagógicas del Ecuador, las Reuniones Científicas INPIN de la Universidad Laica Vicente Rocafuerte de Guayaquil, Ecuador y el Congreso Internacional Virtual “Conectando escuelas” (País Vasco, España).

Dimensión: Investigación

Indicador: Trabajos de titulación de los graduados que guardan relación con la educación superior inclusiva.

El análisis de los temas de los trabajos de titulación con los que se gradúan los nuevos tecnólogos siempre aporta una información valiosa, sobre todo porque permite juzgar acerca de las áreas de mayor interés investigativo y su relación con la solución de los retos que el desarrollo económico, tecnológico y social plantea. 
Al respecto, en relación con la educación superior inclusiva, en la primera sub-etapa no constan trabajos de titulación relacionados con este tema, que comienza a ser de interés de los futuros graduados en la segunda sub-etapa, en la que aparecen dos trabajos: Manual Básico Interactivo de Lengua de Señas para fortalecer la inclusión de la persona con discapacidad auditiva dentro de la familia Institucional del ITF (2016) y Diseño gráfico inclusivo, la interpretación en medios audiovisuales del Instituto Tecnológico de Formación (2017).

Es muy probable que con la presentación y exitosa defensa de los referidos trabajos de titulación se haya comenzado a gestar el surgimiento de una nueva línea de investigación institucional, que con seguridad recibirá un estímulo de incalculable valor con la ampliación de la oferta académica del ITF, en particular, con la próxima apertura de la carrera de Tecnología Superior en Asistencia en Educación Inclusiva.

\subsection{Dimensión: Vinculación con la sociedad.}

Indicador: Proyectos y actividades de vinculación relacionados con las personas con discapacidad.

Durante la primera sub-etapa se desarrolló un proyecto de vinculación relacionado con las personas con discapacidad, dirigido a capacitar en emprendimiento a los padres de familia de la escuela "Luis Felipe Hernández", de la Cooperativa 25 de julio, de la Isla Trinitaria, Guayaquil.

En el transcurso de la segunda sub-etapa el comportamiento de este indicador experimentó un incremento, alcanzando un total de 9 proyectos y actividades de vinculación relacionados con las personas con discapacidad, incluida la realización del diseño pedagógico aplicado a los niños sordos de la escuela particular "Lidia Dean De Henríquez".

Las vivencias y testimonios de los ejecutores y beneficiarios de estos proyectos y actividades, reflejadas en los informes analizados, refuerzan el carácter doblemente inclusivo (Bell, 2017) de la vinculación con la sociedad, evidenciando de esa manera sus amplias posibilidades para el logro de la transformación y la inclusión social.

Dimensión: Vinculación con la sociedad.

Indicador: Estudiantes con discapacidad que realizaron sus prácticas pre-profesionales en el ITF.

Desde el año 2015, es decir, con el comienzo de la segunda sub-etapa, fue posible disponer de informes en los que se constata la realización de las prácticas pre-profesionales en el ITF por 9 estudiantes con discapacidad.

De esta forma el Instituto se convirtió también en un escenario para el cumplimiento de este importante requisito de titulación, lo que de acuerdo con los informes de prácticas pre-profesionales representó un nuevo estímulo para la generación de una cultura organizacional inclusiva y una vía para evaluar de manera directa los resultados de la labor formativa de la institución. 


\section{Conclusiones}

La investigación desarrollada permitió la reflexión acerca de la comprensión y el carácter sistémico de la educación superior inclusiva en relación con los estudiantes con discapacidad y su desarrollo en el ITF como expresión de la respuesta a la necesidad avanzar hacia el cumplimiento de los objetivos de este complejo proceso, completando de esa manera el itinerario delineado en Salamanca. En ese entorno, se constató la diversidad de énfasis y matices contenidos en las diferentes definiciones de educación superior inclusiva que fueron objeto de análisis, corroborando la importancia de continuar trabajando en la conformación de un marco teórico, conceptual y metodológico (Ocampo, 2016) que favorezca una mejor aproximación a la educación superior inclusiva y a sus implicaciones para la práctica.

Al mismo tiempo, se relacionaron distintos ámbitos en cuya transformación se revela el carácter sistémico de la educación superior inclusiva y asociado al mismo, su enorme capacidad potencial para propiciar un verdadero cambio de la educación superior, que ha de estar orientado a enriquecer y concretar la vigencia del mensaje de Salamanca en este nivel educativo.

En ese escenario se enmarca el análisis de la situación de la educación superior inclusiva en Ecuador, donde, como sucede en prácticamente todo el mundo, es apreciable la brecha existente entre los derechos consagrados, incluido el acceso y las posibilidades de formación en la educación superior y los hechos llamados a confirmar su pleno ejercicio.

Desde esa perspectiva, la labor inclusiva que se desarrolla en el ITF en la materialización del cumplimiento de su responsabilidad social, puede ser considerada como un ejemplo de respuesta dirigida a la disminución de la referida brecha, lo que se refleja en el comportamiento de distintos indicadores, que a su vez se convierten en una clara expresión de la indispensable y continua renovación de los propósitos de Salamanca en esta institución.

Como consecuencia de ello, de su identificación y apego a la filosofía educativa proclamada en Salamanca, considerando las necesidades existentes y a raíz de la experiencia inclusiva acumulada en estos años, el ITF presentó y fue aprobado por el Consejo de Educación Superior de Ecuador, el diseño de la carrera de Tecnología Superior en Educación Inclusiva, cuya próxima puesta en marcha representará un nuevo paso de avance en la formación del talento humano que la educación inclusiva demanda.

\section{Referencias}

Acevedo, S. (2015). Perspectivas necesarias sobre educación superior inclusiva con tecnologías de la comunicación en la formación de jóvenes. Revista de Estudios de Juventud, 108, 41-50.

Bell, R. F. (2017). El desarrollo de los procesos sustantivos de la educación superior ecuatoriana ante el reto de la inclusión educativa. Revista Latinoamericana de Educación Inclusiva, 11(1), 199-2 12. https://doi.org/10.4067/S0718-73782017000100012

Escudero, J. M. y Martínez, B. (2011). Educación inclusiva y cambio escolar. Revista Iberoamericana de Educación, 55, 85-105. https://doi.org/10.35362/rie550526

Espinosa, C. X., Gómez, V. G. y Cañedo, C. M. (2012). El acceso y la retención en la educación superior de estudiantes con discapacidad en Ecuador. Formación Universitaria, 5(6), 27-38. https://doi.org/10.4067/SO7 18-50062012000600004 
Esteves, A. y Santos, D. (noviembre, 2014). Una mirada a la inclusión social de las personas con discapacidad: experiencia de Flacso-Ecuador. Comunicación presentada en el III Congreso Internacional MISEAL: Nuevos Desafios para la Inclusión Social y la Equidad en la Educación Superior.

Herdoiza, M. (2015). Construyendo igualdad en la educación superior. Fundamentación y lineamientos para transversalizar los ejes de igualdad y ambiente. Quito: Senescyt/Unesco.

Hernández Fernández, Y. A. y Pérez Véliz, A. P. (2013). Propuesta metodológica para una periodización de las contribuciones de las telecomunicaciones al desarrollo social en Pinar del Río. Avances, 15(4), 440-451.

Loreman, T. (2007). Seven pillars of support for inclusive education: Moving from "why?" to "how?" International Journal of Whole Schooling, 3(2), 22-38.

Maltseva, T. y Boyko, A. (2017). La educación superior inclusiva superior como un nuevo paso en el desarrollo del sistema general de educación superior. Boletín de la Universidad de Omsk, 3, 32-38.

Moriña, A., Cortés, M. D. y Molina, V. M. (2015). Educación inclusiva en la enseñanza superior: Soñando al profesorado ideal. Revista Latinoamericana de Educación Inclusiva, 9(2), 161-175.

Navarros, N. y Luque, M. (2017). La inclusión educativa como responsabilidad social universitaria: Un modelo para la Universidad Metropolitana. RECUS. Revista Electrónica Cooperación Universidad Sociedad, 2(2), 21-24. https://doi.org/10.33936/recus.v2i2.981

Ocampo, A. (2013). Inclusión de estudiantes en situación de discapacidad a la educación superior. Desafíos y oportunidades. Revista Latinoamericana de Educación Inclusiva, 6(2), 227-239.

Ocampo, A. (2016). Experiencias y desafíos sobre educación superior inclusiva. Santiago de Chile: Centro de Estudios Latinoamericanos de Educación Inclusiva.

ONU. (1948). Declaración universal de los derechos humanos. Nueva York, NY: United Nations.

ONU. (2006). Convención sobre los derechos de las personas con discapacidad. Nueva York, NY: United Nations.

Pérez Serrano, E. A. (2015). La educación inclusiva en la educación superior: Relato del contexto universitario cubano. Revista Ibero-Americana de Estudos em Educação, 10 (1), 583-598.

Rubtsova, V. (2018). El desarrollo de la inclusión en la educación superior: Un enfoque de red: Complicación de artículos. Moscú: Biblioteca de la revista Ciencia psicológica y Educación.

Santiesteban, I., Barba Ayala, J. V. y Fernández Álvarez, D. (2017). Inclusión de estudiantes con necesidades educativas especiales en la Universidad Técnica del Norte del Ecuador. Revista Universidad y Sociedad, 9(3), 162-167.

Toscanini, M., Aguilar Guzmán, A. y García Sánchez, R. (2016). Diagnóstico de las políticas públicas de la educación superior en el Ecuador. Revista Cubana de Educación Superior, 35(3), 161-178.

Zinevich, O. V., Degtyareva, V. V. y Degtyareva, T. N. (2016). Educación inclusiva en la educación superior rusa: Desafíos contemporáneos. Власть, 5, 61-67. 


\section{Breve CV de los autores}

\section{Rafael Félix Bell}

Graduado con notas sobresalientes del Instituto Estatal Pedagógico Lenin - Moscú, Rusia (1985), con especialización en Sordopedagogía. Máster en Educación y Máster en Educación Especial (1998). Doctor en Ciencias Pedagógicas por el Instituto Central de Ciencias Pedagógicas, La Habana, Cuba (2009). Profesor auxiliar e investigador acreditado por la Academia de Ciencias de Cuba y SENESCYT (Ecuador). Presidente de Comisión, conferencista magistral y ponente en numerosos eventos científicos nacionales e internacionales. Autor y coautor de más de 70 publicaciones, entre las que se incluyen libros, capítulos de libros, traducciones, ponencias y artículos que han sido publicados en Cuba, México, España, Italia, Chile y Ecuador. ORCID ID: https://orcid.org/0000-00020255-642X. Email: rafael.bell@formacion.edu.ec.

\section{Belinda Marta Lema Cachinell}

Rectora del Instituto Superior Tecnológico de Formación Profesional Administrativa y Comercial, Ingeniera Comercial graduada de la Universidad de Guayaquil, posee un Diplomado Superior en Currículo por Competencias de la Universidad Técnica de Ambato, Magister en Gerencia Educativa de la Universidad Metropolitana y Doctora en Ciencias de la Gestión por la International Caribbean University de Curazao, Doctora en Ciencia Pedagógicas por la Universidad de Oriente de Santiago de Cuba, investigadora acreditada en el Ecuador No. REG-INV-15-00912. Miembro de la red de investigación internacional RED-DEES. Ponente en numerosos congresos nacionales e internacionales y autora de más de veinte artículos científicos. ORCID ID: https://orcid.org/0000-00021403-336X. Email: martalema@formacion.edu.ec.

\section{Emma Zulay Delgado Saeteros}

Vicerrectora Administrativa-Financiera del Instituto Superior Tecnológico de Formación Profesional Administrativa y Comercial, Ingeniera en Sistemas Computacionales graduada de la Universidad Estatal de Milagro en el año 2013. Doctora en Ciencias Pedagógicas en la Universidad de Oriente de Santiago de Cuba, miembro del Consejo editorial de la revista internacional Investigación, Formación y desarrollo: generando productividad institucional ubicada en catálogo LATINDEX, ha recibido varios reconocimientos profesionales entre los que se destacan: "IX Taller Nacional de la Educación Técnica y Profesional con Participación Extranjera”, "Maestro Sociedad 2015”, entre otras. Autora de varios artículos científicos y ponente en numerosos congresos nacionales e internacionales. ORCID ID: https://orcid.org/0000-0003-1432-4885.

Email: zulayd@formacion.edu.ec.

\section{Alejandro Nicolás Lema Cachinell}

Director del Departamento de Investigación del Instituto Superior Tecnológico de Formación Profesional Administrativa y Comercial. Ingeniero en Contaduría Pública y Auditoría CPA y Graduado en la Universidad Estatal de Milagro. Doctor en Ciencias Pedagógicas en la Universidad de Oriente de Santiago de Cuba, director de la revista internacional Investigación, Formación y desarrollo: generando productividad institucional, ubicada en catálogo LATINDEX, revisor externo del volumen 12 no. 1 de 
la Revista Latinoamericana de Educación Inclusiva (RLEI) de la Universidad Central de Santiago de Chile, indexada en SCIELO. Miembro de la red de investigación internacional RED-DEES. Autor de diversas publicaciones y ponente en numerosos congresos nacionales e internacionales. ORCID ID: https://orcid.org/0000-0002-6402-9342.

Email: alejandrol@formacion.edu.ec. 